\title{
IMPACTOS AMBIENTALES Y ENERGÉTICOS DE LAS CIRCUNVALACIONES URBANAS. EL CASO DE LA M-40
}

\author{
'(ENVIRONMENTAL AND ENERGY IMPACTS OF URBAN RING ROADS. \\ THE CASE OF M-40)
}

Andrés Monzón, Dr. Ingeniero de Caminos y $\mathrm{M}^{\mathrm{a}}$ Teresa Alcaide, Ingeniero de Montes

Departamento de Transportes. ETSI Caminos, Canales y Puertos. UPM

Fecha de recepción. 13-X-95

ESPAÑA

$113-39$

RESUMEN

En el presente artículo se exponen aspectos generales acerca de algunos impactos derivados de la construcción de circunvalaciones urbanas. Se detalla también brevemente la metodología seguida en la cuantificación de algunos de los efectos ambientales y energéticos derivados de la apertura del tramo Noroeste de la circunvalación a Madrid "M-40".

\section{SUMMARY}

This article presents general aspects of some of the impacts produced by the construction of urban bypasses. A brief description is also given of the methodology followed in the quantification of some environmental and energy impacts that arose from the opening to traffic of the Northwest stretch of the Madrid M-40 ring road.

\section{Introducción}

A menudo los impactos producidos por la construcción de una carretera sobre los distintos factores del medio se consideran negativos, porque se tiende, quizás, a confundir medio con medio natural, olvidando otros factores igualmente importantes de tipo social, económico, etc.

En el caso concreto de las circunvalaciones, los efectos sobre la población suelen ser benefíciosos, por modificar la distribución del tráfico, de modo que en el interior de la ciudad disminuye el tráfico de paso. Corresponde al planificador estudiar si estos beneficios tienen mayor peso que los costes sociales y económicos. Por dicha razón, este tipo de actuaciones han de considerarse en el plano más amplio de la planificación territorial con el fin de considerar todos los efectos posibles.

Por otra parte, los efectos son difíciles de localizar, pero mucho más complejo es tratar de cuantificarlos en térmi- nos monetarios. Si esto fuese posible se facilitaría enormemente la posibilidad de comparación entre trazados alternativos.

\section{Los cinturones de circunvalación}

Como es sabido, los cinturones o rondas urbanas son todas aquellas carreteras o vías urbanas que cumplen funciones de circunvalación para los movimientos de paso que atraviesan la ciudad. Sin embargo, no sólo el tráfico de paso utiliza este viario sino que también el tráfico urbano de penetración puede utilizar la circunvalación para desplazarse de una parte de la ciudad a otra.

Las funciones que desempeña dependerán principalmente de su localización con respecto a la ciudad. Estas funciones pueden ser de circunvalación y de conexión (tanto de la malla viaria urbana con las variantes exteriores, como de las diversas vías radiales entre sí); también pueden actuar como distribuidoras del tráfico e incluso a veces 
tienen la función de contener o limitar la expansión de la zona urbanizable, y de dar homogeneidad al itinerario de los vehículos.

\section{Efectos de las circunvalaciones sobre la movilidad urbana}

Durante las últimas décadas, el aumento de población, debido sobre todo al fuerte desarrollo económico de las principales regiones metropolitanas, se ha unido a un gran aumento de los viajes al trabajo. Añadiendo a esto el incremento en el índice de motorización, es fácil explicar el notable aumento de la movilidad, con los consiguientes problemas de tráfico. Resulta entonces necesario descargar las calles centrales de las ciudades para lo cual deben existir vías exteriores que puedan recibir el tráfico que se transfiere desde el centro, a la vez que se realiza un análisis de las medidas de regulación de tráfico en la red interna de la ciudad.

La construcción de las nuevas infraestructuras produce una modificación en la distribución del tráfico en la red, pero también pueden producirse efectos no esperados; incluso puede suceder que empeoren las condiciones de ciertas vías. En el caso de la construcción de circunvalaciones, no es infrecuente que el porcentaje de vehículos que sigue atravesando el centro urbano tras la puesta en servicio de la circunvalación sea importante, ya que el cinturón puede suponer un rodeo que alargue el itinerario, de forma que el recorrido por el centro de la ciudad sea una alternativa interesante, especialmente fuera de las horas punta. Por lo tanto, es necesario que la circunvalación permita un ahorro en tiempo para que el usuario cambie de itinerario, o bien que mejore claramente la comodidad del trayecto con tiempos no muy superiores.

Por otra parte, los cinturones de circunvalación juegan un papel muy importante desde el punto de vista de la accesibilidad a las diferentes zonas de la ciudad: éstos deben comunicar, por un lado, las áreas periféricas con los espacios centrales y, por otro, deben facilitar las relaciones entre distintos espacios periféricos donde los movimientos transversales y el uso del vehículo privado predominan.

\section{Efectos medioambientales y energéticos}

Los efectos de tipo medioambiental derivados de la construcción y funcionamiento de una circunvalación pueden ser numerosos, pues incide en muchos componentes del medio, tanto físico como biótico y social. La importancia de unos y otros puede ser distinta dependiendo de cada caso concreto. Hay componentes ambientales afectados a cuyos impactos es viable aplicar medidas correctoras; sin embargo en los de tipo social estas medidas suelen escapar a las posibilidades del proyecto.
Los impactos derivados de la circunvalación pueden ser clasificados como se detalla a continuación

1) efectos medioambientales propiamente dichos

2) efectos energéticos y económicos

3) efectos sociales

\section{Efectos medioambientales}

Aparte de los impactos que pueden producirse sobre la flora, fauna o vegetación, estudiados ampliamente por los especialistas, existen otros efectos ambientales como los siguientes:

El aumento de la contaminación atmosférica, que en las ciudades es debida en gran parte al tráfico rodado, viéndose agravada en condiciones de altas intensidades de tráfico y congestión junto con situaciones de estabilidad meteorológica. La cantidad de emisiones dependerá de la velocidad y régimen de circulación, existiendo para cada tipo de vehículo (ligero, pesado) una velocidad en la que la cantidad de gases emitidos es mínima.

Por otra parte, conocidos los niveles de emisión en las vías de circulación, debe estudiarse la concentración de gases contaminantes en cualquier otro punto de la ciudad, pues son los altos niveles de concentración los que afectan a la salud de la población y causan daños sobre los materiales y la vegetación. La concentración dependerá de cómo se dispersen los gases emitidos y cuanto más alejadas estén las fuentes de emisión, menos afectará a la población residente, de ahí la conveniencia de que las circuvalaciones circulen por zonas no pobladas.

. La contaminación acústica, de la cual el tráfico rodado es responsable entre otras fuentes, también ocasiona perjuicios a la salud de los ciudadanos más expuestos. En los últimos años se han instalado pantallas acústicas para corregir este impacto, pues las normativas en materia de ruido han ido disminuyendo los niveles permitidos en la U.E.

. El deterioro de los elementos del patrimonio históricoartístico próximos a las ciudades o situados en ellas, que sufren los efectos corrosivos de los contaminantes emitidos por los vehículos, y cuyos costes de reparación y mantenimiento son importantes.

\section{Efectos energético-económicos}

Algunos de los efectos energéticos y económicos pueden ser los siguientes:

. Un gasto de recursos energéticos no renovables: gasto de combustible y lubricante de los vehículos que circulan por vías congestionadas y que con la construcción de circunvalaciones supondrá un ahorro. 
La pérdida de tiempo de viaje derivado del descenso de la velocidad media de circulación en el interior de las ciudades (congestión); las circunvalaciones también contribuyen a solucionar este aspecto.

.Los accidentes generados por el aumento de la motorización, que ocasionan un coste social cuantificable por concepto de pérdida de vidas humanas, atención sanitaria, pérdida de horas laborables y otros efectos no cuantificables

\section{Efectos sociales}

Los efectos sociales son numerosos y no se analizarán aquí, pero se resumen y traducen en el deterioro de la calidad de vida en las ciudades, término en el que están consideradas en su conjunto todas las molestias que los habitantes de las ciudades deben soportar diariamente, como la contaminación atmosférica y acústica, ya mencionadas, el impacto visual provocado por miles de vehículos, los problemas debidos a la pérdida de tiempo en los desplazamientos, el efecto barrera provocado por las grandes avenidas y carreteras urbanas, etc.

\section{La circunvalación a Madrid}

El planeamiento urbano de Madrid, se puede decir que comienza con el Plan de Ensanche de 1860 (Plan de Castro) y desde entonces el crecimiento de la ciudad fue planteando la necesidad de cinturones.

En la década de los sesenta se consolidaron los proyectos de la red viaria con un sentido descentralizador, planteando una red nacional y otra comarcal. El olvido de los anillos periféricos se puso de manifiesto en la congestión de las rondas madrileñas, que se veían obligadas a recoger todo el tráfico exterior. Para resolver este problema, el Ayuntamiento de Madrid emprendió la construcción de una serie de pasos elevados sobre ellas.

La M-30, primera vía arterial importante de carácter perimetral, aunque no proporcionaba un circuito completo, no se abrió a la circulación hasta 1974. Ya en su inauguraciónera excesivamente interna al perímetrourbano, y su función era distribuir el tráfico de penetración al centro. En aquellos momentos tuvo lugar un considerable aumentode la movilidad en todas las ciudades españolas.

Actualmente la M-30 es una autovía urbana en todo su trazado excepto en el primer tramo de la Avenida de la Ilustración, en el que existen cruces a nivel. Con una longitud de $32 \mathrm{~km}$, es la circunvalación que limita la llamada almendra central, y la principal vía distribuidora del tráfico interior de la capital. En el Cuadro 1 se muestra la evolución del tráfico en la citada vía y en en interior de la misma.

El cinturón M-40, todavía no completo a falta del tramo entre nacional VI y nacional I, además de canalizador del tráfico de paso, es una vía colectora/distribuidora en los movimientos entre la periferia y el centro. En sus márgenes se sitúan cada vez más asentamientos urbanos. Influye en la dinámica regional estructurando el crecimiento y la desconcentración de actividades a corto plazo.

\section{Cuadro 1}

\section{Características de la movilidad en Madrid}

\begin{tabular}{|c|c|c|c|c|c|}
\hline \multirow[t]{2}{*}{ Año } & \multicolumn{2}{|c|}{$\begin{array}{c}\text { Datos de circulación en } \\
\text { el interior de la } \\
\text { circunvalación } M-30\end{array}$} & \multicolumn{3}{|c|}{$M-30$} \\
\hline & IMD (veh/día) & $\begin{array}{c}\text { Velocidad media } \\
(\mathrm{km} / \mathrm{h})\end{array}$ & $\begin{array}{l}\text { IMD } \\
\text { (E-99) }\end{array}$ & \%pesados & $\begin{array}{c}\text { Velocidad } \\
\text { media }(\mathrm{km} / \mathrm{h})\end{array}$ \\
\hline 1987 & 586.231 & 23,16 & 202.254 & 6 & 57,09 \\
\hline 1988 & 581.419 & 22,37 & 200.698 & 7 & 54,66 \\
\hline 1989 & 590.944 & 22,60 & 229.047 & 8 & 50,91 \\
\hline 1990 & 602.878 & 23,10 & 234.888 & 7 & 51,28 \\
\hline 1991 & 614.796 & 22,76 & 224.748 & 6 & 61,79 \\
\hline 1992 & 631.567 & 23,76 & 223.040 & 3 & 70,85 \\
\hline
\end{tabular}




\section{Un caso particular: el tramo Noroeste de la M-40}

En este artículo se resume un estudio realizado en la Universidad Politécnica de Madrid, en el que se realizó una evaluación de los efectos económico-sociales y ambientales de la circunvalación a Madrid M-40. En el que se trataron de comparar los efectos anteriores y posteriores a la construcción de su tramo Noroeste, el comprendido entre Alcorcón $(\mathrm{N}-\mathrm{V})$ y el nudo de Hortaleza (N-I).

La metodología consistió en la modelización del tráfico tomando como base los datos de aforos del año 1993, calculando las velocidades medias y localizando las zonas geográficas por las que los vehículos circularían en la situación futura, cuando el tramo en estudio estuviese en servicio.

La red de Madrid se representó mediante un grafo simplificado (Figura 1), clasificado en 16 tipos de vías distintos, de acuerdo a sus características geométricas, capacidad, regulación semafórica, limitaciones legales a la velocidad, etc. de cada uno.

Se utilizaron como datos de partida las intensidades de tráfico, porcentaje de vehículos pesados y velocidades medias de circulación para un día laborable medio, en cada uno de los tramos de la red considerada.
Mediante la información anterior se pudo calcular el total de litros de combustible consumido, utilizando fórmulas que dependen de la velocidad de circulación, pendiente, tipo de tramo (urbano, no urbano) y tipo de vehículo (turismo, camión) (MOPU, 1992). Como ejemplo, se presenta el consumo para turismos en zona urbana y camiones en tramo recto y llano (Fig.2).

La intensidad de tráfico y velocidad media de circulación en cada segmento de la red en la situación "SIN tramo Noroeste de la M-40" corresponde a 1995, suponiendo un aumento de tráfico global de 5\% anual desde 1992 (último mapa de tráfico realizado por el Ayuntamiento de Madrid). Para el escenario futuro ( $C O N$ dicho tramo completo) los datos necesarios se determinaron a partir de la modelización y reasignación de tráfico obtenida por el programa TRIPS; este sistema asigna las cargas de tráfico de la matriz origen-destino buscando el coste mínimo del transporte en el conjunto de toda la red. Posteriormente, los resultados en los principales segmentos fueron verificados con datos reales proporcionados por el Área de Circulación y Transportes del Ayuntamiento de Madrid.

Como datos iniciales, necesarios para el sistema TRIPS, y tomando como base los estudios realizados por el Ministerio de Obras Públicas (MOPU, 1992), se calculó la presión teórica total de movimientos de penetración en el tramo en

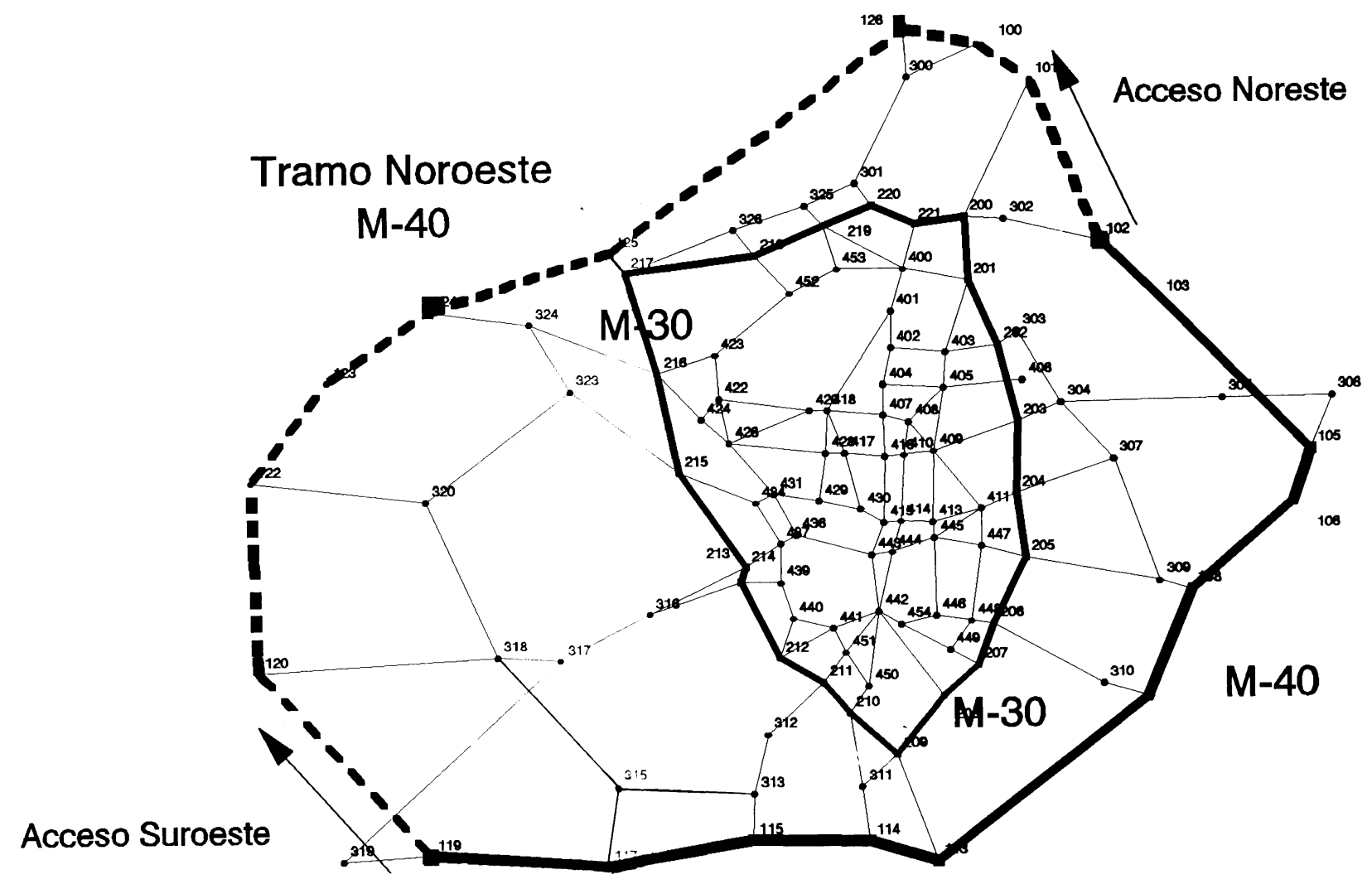

Fig. 1.- Grafo simplificado de la red considerada. 

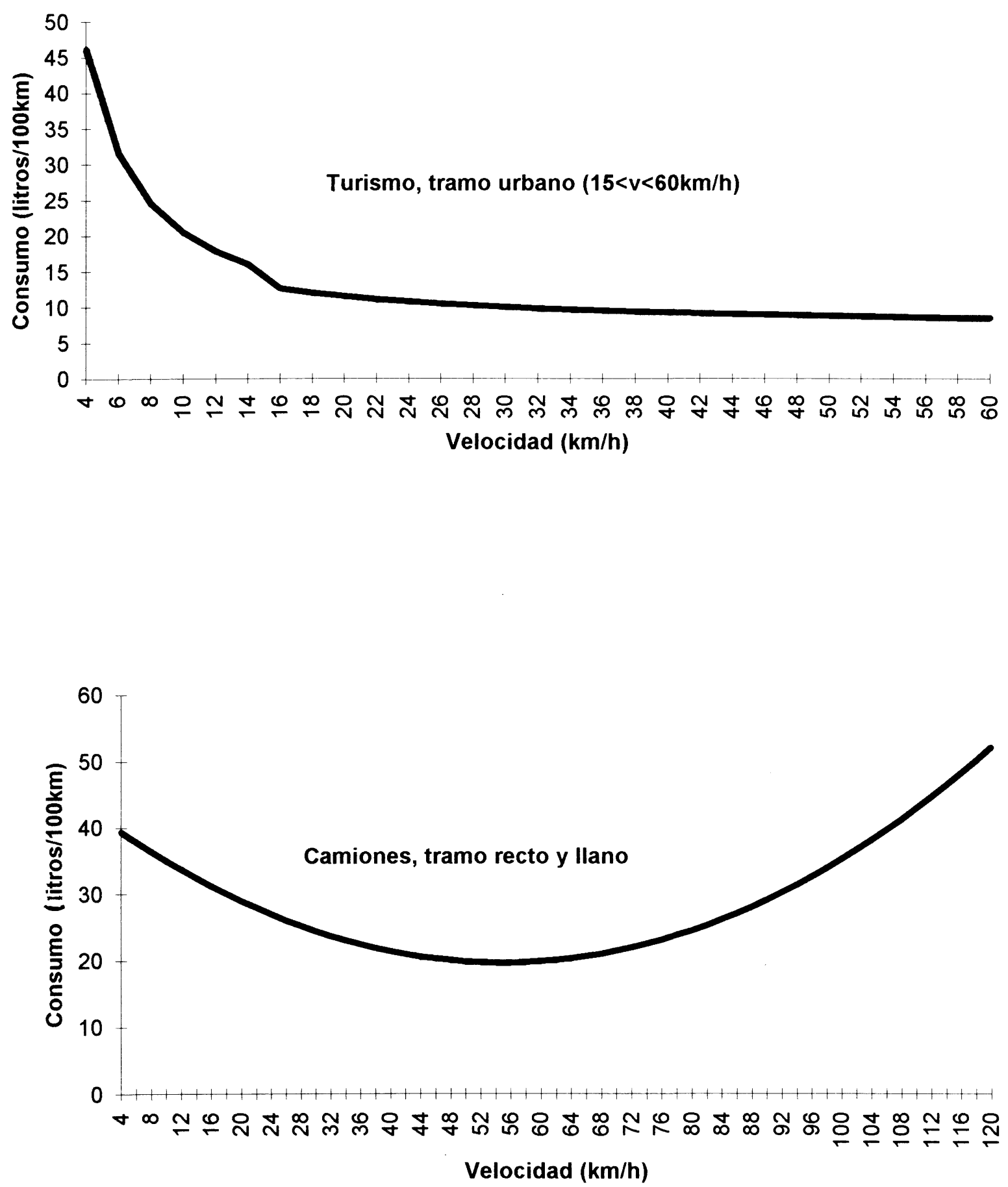

Fig. 2.- Consumos de combustible.

estudio; ésta sería, en la hipótesis máxima, de unos 43.000 veh/día en el acceso Suroeste y de 72.000 veh/día en el acceso Noreste (ver Fig. 1). Parte de esta presión utilizará las vías de circunvalación del Noroeste y parte entrará o saldrá por viario secundario, por lo que puede deducirse la distinta localización de los problemas ambientales.

Evidentemente, de la exactitud del modelo de tráfico dependerá la correcta evaluación de impactos, pues, en definitiva, éstos dependen de la cantidad de vehículos y combustible consumido.

\section{Cuantificación de los efectos}

Aunque todos los efectos mencionados son consecuencia directa del tráfico $\mathrm{y}$, por lo tanto, se generan por la construcción de una circunvalación, no todos pueden ser medidos sin caer en un alto grado de subjetividad, a menos que se disponga de una gran cantidad de información.

Por lo tanto, para estimar los efectos que ocasiona el cierre Noroeste de la circunvalación a Madrid M-40, sólo se consideraron los que pudieron ser deducidos a partir de 
datos medidos o de los que estaban disponibles, tanto en los centros de documentación como en el Ayuntamiento y MOPTMA. Estos datos básicos son las intensidades medias diarias, longitudes y velocidad media de circulación. Los efectos incluidos en esta evaluación fueron:

- los costes de funcionamiento (combustible, lubricante)

- la contaminación atmosférica

- el aumento del tiempo de viaje

\section{Costes de funcionamiento}

Los costes de funcionamiento de los vehículos que están afectados directamente por las condiciones de circulación son los costes de tracción. De ellos consideraremos el consumo de combustible y el consumo de lubricantes.

\section{Consumo de combustible}

En los proyectos y estudios de carreteras españoles, el consumo de combustible se calcula basándose en las recomendaciones del Ministerio de Obras Públicas(MOPU, 1992) para la evaluación económica de proyectos de carreteras. El consumo dependerá del tipo de vehículo (simplificando, serán turismos o camiones) y de las características del tramo recorrido: velocidad media, pendiente, tramo urbano o carretera.

Las fórmulas empleadas se detallan en el Cuadro 2.

\section{Consumo de lubricantes}

El cálculo del consumo de lubricantes representa un porcentaje del de combustible. El MOPU (1992) asigna un valor de $1,2 \%$, pero según un estudio de Bertrand (1978) se puede establecer en un $1,6 \%$ para el caso de los turismos.
El primero, aunque es representativo del parque automovilístico español, se habasadoen ensayos realizados en carretera a velocidad constante, por lo que no es muy representativo de las condiciones de circulación urbana, mientras que el estudio de Bertrand, aunque sí es representativo de estas condiciones, no lo es del parque automovilístico español, por lo que mientras no se realicen nuevos estudios que determinen un porcentaje de consumo de lubricante representativo para España, en condiciones de circulación urbana, se podría tomar el valor promedio entre los estudios antes señalados, es decir, un porcentaje igual al $\mathbf{1 , 4 \%}$.

De esta forma obtendremos el consumo de lubricante $\left(C_{L}\right)$ mediante la siguiente expresión:

$$
\mathrm{C}_{\mathrm{L}}=0,014 \cdot \mathrm{C}_{\mathrm{C}}
$$

\section{Niveles de contaminación}

En cuanto a la cantidad de contaminantes emitidos por los vehículos, existen numerosos estudios al respecto. Entre éstos se pueden mencionar el inventario realizado por el programa CORINAIR de la CE (DG XI), que presenta los indices de emisión para el parque automotriz europeo (Cuadro 3). Este trabajo está realizado por desagregación de cada tipo de contaminante: partículas, óxidos de azufre, óxidos de nitrógeno, monóxido de carbono y componentes orgánicos volátiles (COV, que incluye hidrocarburos no quemados y plomo tetraetilo).

Otro estudio es el proporcionado por Carrasco (1992) para el caso específico de España, el cual presenta los resultados siguientes, en gramos de contaminante por litro de combustible consumido (Cuadro 4).

\section{Cuadro 2}

\section{Cálculo del consumo de combustible}

\begin{tabular}{|c|c|c|}
\hline Tipo de tramo & Turismos & Vehículos pesados \\
\hline Recto y llano & $C_{C}=91,86-0,98 v+7,08 \cdot 10^{-3} v^{2}$ & $C_{c}=425,65-8,35 v+7,61 \cdot 10^{-2} v^{2}$ \\
\hline Recto con rampas & $\begin{array}{l}C_{c}=91,86-0,98 v+7,08 \cdot 10^{-3} v^{2}++p \\
\left(24,09-0,47 v+4,74 \cdot 10^{-3} v^{2}\right)\end{array}$ & $\begin{array}{l}C_{c}=388,18-7,32 v+7 \cdot 10^{-2} v^{2}+p \\
\left(101,28+1,99 \cdot 10^{-2} v+7,85 \cdot 10^{-3} v^{2}\right.\end{array}$ \\
\hline Urbanos & $C_{C}=(70+90 / v)$ para $15<v<60 \mathrm{~km} / \mathrm{h}$ & $C_{c}=(70+90 / v)$ para $15<v<60 \mathrm{~km} / \mathrm{h}$ \\
\hline
\end{tabular}

donde:

$\mathrm{C}_{\mathrm{C}}=$ consumo en $\mathrm{cm}^{3} / \mathrm{km}$

$v=$ velocidad del tramo en $\mathrm{km} / \mathrm{h}$

$p=$ pendiente en $\%$ (positivo en rampas, negativo en pendientes) 


\section{Cuadro 3}

Factor de emisión de contaminantes para el sector transporte programa CORINAIR de la CE (DG XI)

\begin{tabular}{|c|c|c|c|c|c|}
\hline \multirow{2}{*}{$\begin{array}{l}\text { Tipo de } \\
\text { vehículo }\end{array}$} & \multirow{2}{*}{$\begin{array}{l}\text { Pauta de } \\
\text { Conducción }\end{array}$} & \multicolumn{4}{|c|}{ Contaminante $(\mathrm{g} / \mathrm{km})$} \\
\hline & & Partículas & $\mathrm{NO}_{x}$ & co & $\mathrm{SO}_{2}$ \\
\hline \multirow{3}{*}{$\begin{array}{l}\text { Vehículo } \\
\text { Ligero } \\
\text { Gasolina }\end{array}$} & Urbana & 0,00 & 1,62 & 17,00 & 1,87 \\
\hline & Rural & 0,00 & 2,23 & 6,00 & 1,87 \\
\hline & Interurbana & 0,00 & 2,67 & 3,00 & 1,87 \\
\hline \multirow{3}{*}{$\begin{array}{l}\text { Vehículo } \\
\text { Ligero } \\
\text { Diesel }\end{array}$} & Urbana & 0,20 & 0,70 & 1,00 & 5,94 \\
\hline & Rural & 0,15 & 0,55 & 0,85 & 5,94 \\
\hline & Interurbana & 0,30 & 1,00 & 1,25 & 5,94 \\
\hline \multirow{3}{*}{$\begin{array}{l}\text { Vehículo } \\
\text { Pesado } \\
\text { Diesel }\end{array}$} & Urbana & 1,60 & 18,20 & 7,30 & 5,94 \\
\hline & Rural & 1,60 & 24,10 & 3,70 & 5,94 \\
\hline & Interurbana & 1,30 & 19,80 & 3,10 & 5,94 \\
\hline
\end{tabular}

Fuente: Proyecto PECAM (1991)

\section{Cuadro 4}

Índices de emisión para vehículos en España

\begin{tabular}{||l|c|c|c|c|c|c||}
\hline \multirow{2}{*}{ Vehículo } & \multicolumn{7}{|c|}{ gramos/litro } \\
\cline { 2 - 7 } & $\mathrm{CO}$ & $\mathrm{CO}_{2}$ & $\mathrm{HC}$ & $\mathrm{NO}_{x}$ & Part. & $\mathrm{SO}_{2}$ \\
\hline \hline Gasolina & 360,0 & $1.821^{*}$ & 30,0 & 15,3 & 1,0 & 1,4 \\
\hline Diesel & 7,0 & & 10,0 & 19,0 & 30,0 & 8,3 \\
\hline
\end{tabular}

Fuente: Carrasco, C. et al. 1992

* Dato proporcionado por REPSOL, S.A.

En este estudio no se mencionan datos de $\mathrm{CO}_{2}$, pero de acuerdo con la información proporcionada por estudios hechos en Repsol S.A., se ha considerado que se emite la cantidad de 1.821 gramos de este contaminante por cada litro de gasolina consumida.

Estos últimos valores fueron empleados en el caso que nos ocupa, con los que, a partir de los consumos de combustible, se pueden estimar las emisiones de los vehículos en distintas condiciones de circulación para el caso del parque automotriz sin más que multiplicar el consumo por la cantidad de contaminantes que genera la combustión de cada litro.

La predicción de la cantidad de gases contaminantes emitidos a la atmósfera es útil para establecer los niveles de concentración de los mismos, para lo cual es necesario conocer la forma y velocidad de dispersión. La forma de abordar este paso fue a través de una modelización teórica.

Los modelos de difusión de contaminantes atmosféricos son instrumentos válidos para estimar el comportamiento de los contaminantes en el aire mediante la formulación matemática de los fenómenos que tienen lugar (Pasquille, 1982). Son muchas las áreas rurales y urbanas donde se emplean con éxito como herramienta de trabajo habitual para estudiar y predecir los problemas de contaminación existentes (McCrae, 1988).

Laúnica dificultad que presenta su utilización es la necesidad de determinar los valores de los coeficientes de dispersión 
y su variación en función del tipo de estabilidad atmosférica y de su distancia al foco emisor. La determinación de estos coeficientes se realiza siempre experimentalmente y de la bondad de su elección dependen en gran medida los resultados obtenidos.

Para el área de Madrid se eligió un modelo de tipo gaussiano, suponiendo una fuente de emisión lineal e infinita. La expresión que toma la ecuación básica de difusión se resume en la siguiente:

$$
\mathbf{C}=\mathbf{N} \cdot \mathbf{Q} \cdot \mathbf{F}
$$

donde

$\mathrm{C}=$ Concentracion $\left(\mathrm{g} / \mathrm{m}^{3}\right)$ del contaminante considerado

$\mathrm{N}=$ flujo de vehículos (vehículos $/ \mathrm{h}$ ).

$\mathrm{Q}=$ emisión de contaminantes ( $\mathrm{g} / \mathrm{veh} .-\mathrm{km})$.

$\mathrm{F}=$ factor de dispersión $\left(\mathrm{sg} / \mathrm{m}^{2}\right)$, que toma la forma siguiente en las condiciones de este estudio:

$\mathrm{F}=1 /\left(3,6 \cdot 10^{6} \mathrm{u} \cdot \sigma_{\mathrm{z}}\right)$, siendo:

$\mathrm{u}=$ Velocidad efectiva del viento $(\mathrm{m} / \mathrm{s})$

$\sigma_{z}=$ Coeficiente gaussiano de dispersión vertical (m).

Se utilizó un coeficiente de dispersión del tipo sugerido por Pasquille y Smith (1982), que resulta ser de la forma siguiente:

$$
\sigma_{z}=a \cdot x^{b}
$$

Los valores concretos de los coeficientes a y b dependen básicamente de los datos climatológicos del punto de aplicación: temperatura, presión atmosférica, humedad relativa del aire, velocidad de viento, etc. Todos estos datos son medidos en un conjunto de 24 estaciones pertenecientes a la Red de Control de Contaminación del Ayuntamiento de Madrid, distribuidas por toda el área urbana.

La ciudad de Madrid suele presentar problemas graves sólo cuando se producen episodios de gran estabilidad atmosférica -frecuentes en las épocas invernales-. Durante dichos períodos pueden llegar a alcanzarse los valores límite establecidos para alguno de los contaminantes en la legislación municipal y europea vigente (por ejemplo para los óxidos de nitrógeno). Por esta razón se propuso utilizar una velocidad de viento efectiva de $1 \mathrm{~m} / \mathrm{s}$, homogénea en todo el área considerada, que puede representar, con bastante aproximación, la situación correspondiente a las épocas en que se producen las mayores dificultades.

La determinación del flujo de vehículos, N, y el cálculo de la velocidad de emisión de contaminantes, $\mathrm{Q}$, se han obtenido mediante la utilización del programa de asignación de tráfico TRIPS, mencionado más arriba. La localización geográfica de los niveles de contaminación atmosférica se determinó aplicando el modelo mediante un sistema de información geográfica (S.I.G.) de tipo celda o ráster, en el que se introdujeron los datos básicos.
El modelo de dispersión, una vez particularizadas todas las variables para el caso de Madrid, se puede resumir por medio de una expresión como la siguiente:

$$
\mathrm{Ci}\left(\mathrm{mg} / \mathrm{m}^{3}\right)=1,2826 \cdot 10^{-6} \cdot \mathrm{IMD}_{\mathrm{j}} \cdot \mathrm{L}_{\mathrm{j}} \cdot \mathrm{E}_{\mathrm{ij}} \cdot \mathrm{x}^{-0.40}
$$

donde:

$\mathrm{Ci}=$ concentración del contaminante $\mathrm{i}$.

$\mathrm{IMD}_{\mathrm{j}}=$ intensidad media diaria de vehículos para el tramo $\mathrm{j}$ en vehículos/dia-km.

$\mathrm{L}_{\mathrm{j}}=$ longitud del tramo $\mathrm{j}$ en $\mathrm{km}$.

$\mathrm{E}_{\mathrm{ij}}=$ emisión del contaminante $\mathrm{i}$ en el tramo $\mathrm{j}$, en $\mathrm{kg} /$ día$\mathrm{km}$.

$\mathrm{x}=$ distancia, en $\mathrm{m}$, de cada punto del mapa a la fuente contaminante (vía de comunicación) más próxima.

El proceso de cálculo, que se hizo para cada uno de los tramos, superponiendo posteriormente los resultados de todos ellos, comenzó por la asignación de la intensidad de tráfico, así como las longitudes y cantidades emitidas de cada contaminante correspondientes a cada uno de los tramos digitalizados.

Este proceso, realizado para los dos casos considerados ( $\sin$ y con M-40 Noroeste), permitió comparar y localizar los niveles de contaminación y evaluar el impacto sobre el factor del medio calidad del aire antes y después del cierre de la M-40. Se presenta como ejemplo la Figura 3 correspondiente a la concentración de $\mathrm{CO}$ en el caso de la situación "con M-40 Noroeste".

\section{Tiempo de viaje}

Una de las características de una circunvalación bien planificada consiste en el ahorro en el tiempo de viaje de los usuarios. El cálculo de este parámetro en cada uno de los segmentos considerados de la red se realiza a partir de la velocidad media de circulación, velocidad de referencia (situación ideal sin congestión), longitud del segmento y volumen de tráfico soportado por el tramo (Villanueva, 1994).

De esta forma, los efectos totales diarios se calcularon para un día laborable medio. Los beneficios anuales se cuantificaron mediante una extrapolación de los resultados diarios al año completo; de acuerdo a los estudios llevados a cabo en Madrid sobre la participación del tráfico en los fines de semana en el total anual (INECO, 1989), se aplicó un factor de expansión de 319. Este factor determinó en función de las diferencias horarias y de volumen global de tráfico entre los fines de semana y el día laborable medio, basándose en la intensidad media diaria y velocidad real en los días correspondientes a los fines de semana y festivos. 

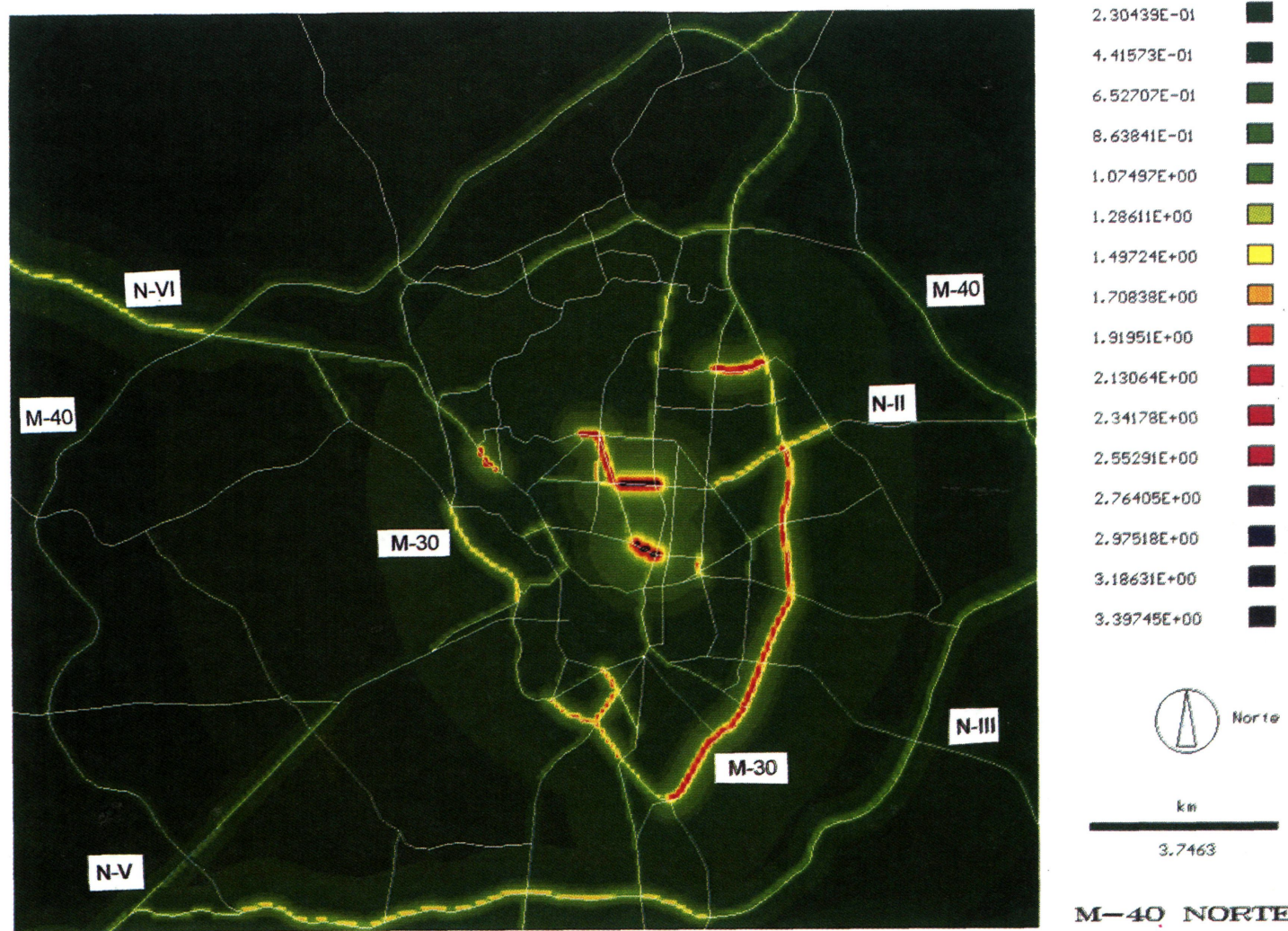

Fig. 3.- Concentración de CO esperada según modelo de difusión.

\section{Resultados}

Una vez calculados los impactos en las dos situaciones -antes y después del proyecto-, se hizo la comparación por diferencia entre ambas. El resultado es el balance que se presenta en el Cuadro 5, en el que se recogen las cifras finales del estudio. Las cifras negativas corresponden a un efecto negativo y las positivas a un beneficio.

\section{Cuadro 5}

Beneficios anuales. Diferencia entre la situación SIN y CON cierre Noroeste

\begin{tabular}{|c|c|c|c|c|c|c|c|c|c|c|c|c|c|}
\hline \multicolumn{3}{|c|}{$\begin{array}{c}\text { Datos básicos. Total Red } \\
\text { Considerada }\end{array}$} & \multirow{2}{*}{$\begin{array}{l}\text { Tráfico } \\
\text { miles de } \\
\text { veh-km }\end{array}$} & \multicolumn{2}{|c|}{$\begin{array}{l}\text { Consumos } \\
10^{3} \text { litros }\end{array}$} & \multicolumn{7}{|c|}{ Emisiones (t.) } & \multirow{2}{*}{$\begin{array}{l}\text { Tiempo } \\
\text { viaje } \\
10^{3} \\
\text { horas }\end{array}$} \\
\hline Zona & $\begin{array}{c}\text { Longitud } \\
(\mathrm{km})\end{array}$ & $\begin{array}{c}\text { Velocidad } \\
(\mathrm{km})\end{array}$ & & Combust. & Lubric. & co & $\mathrm{CO}_{2}$ & $\mathrm{HC}$ & $\mathrm{NO}_{x}$ & $\mathrm{SO}_{2}$ & $\mathrm{~Pb}$ & Partic. & \\
\hline$M-40$ & 71.98 & $-11,81$ & .684 .357 & -81.077 & .901 & .29 .188 & -147.842 & -2.432 & -1.218 & .114 & -9.93 & .958 & .6 .551 \\
\hline$M-30$ & 33,00 & $-6,41$ & 147.589 & 27.352 & 300 & 9.847 & $49.808^{\circ}$ & 821 & 410 & 38 & & & 8.570 \\
\hline $\begin{array}{l}\text { Interior } \\
\mathrm{M}-30\end{array}$ & 92.83 & $-1,38$ & 37.841 & 28.911 & 405 & 10.408 & 52.847 & 887 & 434 & 40 & 11.98 & 758 & 23.997 \\
\hline $\begin{array}{l}\text { Entre } \\
M-30 y \\
M-40\end{array}$ & 129.32 & $\cdot-7.22$ & 376.446 & 47.252 & 596 & 17.011 & 88.047 & 1.418 & 709 & 68 & & & 15.834 \\
\hline Total & 327,13 & $-7,60$ & .122 .480 & 22.438 & 400 & 8.078 & 40.869 & 673 & 337 & 31 & 2,03 & .200 & 41.660 \\
\hline
\end{tabular}

Valores en unidades emitidas o consumidas en un año 
A la vista del citado cuadro se puede concluir que el cierre Norte de la carretera de circunvalación M-40 resultará claramente beneficioso para el conjunto del Área Metropolitana de Madrid, desde el punto de vista de los impactos considerados. El cambio en los flujos de tráfico yen lavelocidades produce unos resultados más equilibrados, tanto de emisiones como de consumo energético, ya que mejora la distribución del tráfico y de los efectos asociados a él.

El beneficio puede resumirse en los siguientes valores:

Emisión de contaminantes atmosféricos

En conjunto, habrá una reducción anual de emisiones de contaminantes, a pesar de que aumenta ligeramente el tráfico:

- 8.078 toneladas menos de $\mathrm{CO}$, que supone un $1,6 \%$ sobre el total emitido

- 40.859 toneladas menos de $\mathrm{CO}_{2}$ (un 1,75\%)

- 673 toneladas menos de $\mathrm{HC}$ (un 1,5\%)

- 337 toneladas menos de $\mathrm{NO}_{\mathrm{x}}$ (un 2,0\%)

- 31 toneladas menos de $\mathrm{SO}_{2}$ (un 1,0\%)

Consumo energético

La reducción anual del consumo de combustible y lubricante es la siguiente:
- 22,4 millones de litros de combustible (1,1\% de ahorro) - 400.000 litros de lubricante $(0,9 \%)$

\section{Ahorro de tiempo}

El ahorro de tiempo, que supone el mayor impacto positivo de todos los estudiados, se cifra en 41,6 millones de horas anuales, un $\mathbf{7 \%}$ de ahorro.

Por otra parte, la utilización de un sistema de información geográfica permitó localizar geográficamente las áreas donde se producirían los niveles de reducción más importantes (Fig. 4) ${ }^{1}$. De la superposición de esta información con los datos de población, se pudo establecer qué porcentaje de ésta podría ser beneficiada por la apertura del nuevo tramo y dónde residía. Así, la población expuesta a los niveles más bajos de concentración de contaminantes es de un $64 \%$ más que en la situación inicial, existiendo una disminución de la cantidad de personas que soportan niveles altos: de 527.000 pasan a ser 865.000 personas

Las zonas donde se localizan estas reducciones se sitúan principalmente en zonas pobladas: Sinesio Delgado (M-30), inmediaciones de la M-30 Sur y Este y, sobre todo, en las zonas residenciales situadas junto al tramo de la N-VI que discurre entre la M-40 y la Carretera de Castilla, en el distrito de Moncloa.
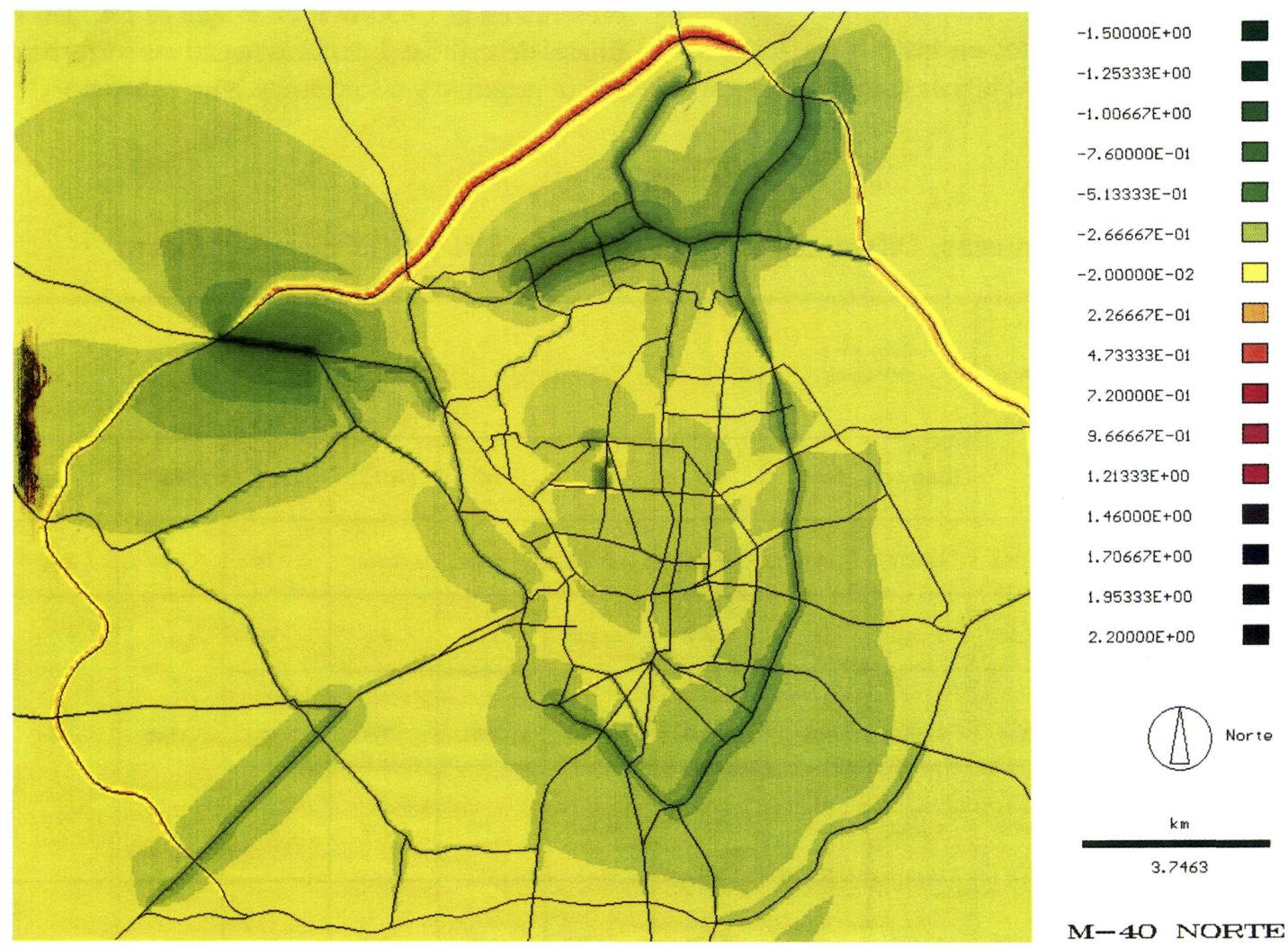

Fig. 4.- Diferencia de concentración de $\mathrm{CO}$ (negativo equivale a disminución).

\footnotetext{
${ }^{1}$ Se hace notar que tanto la Figura 3 como la 4 corresponden únicamente a la contaminación debida al tráfico.
} 
Cabe destacar que la reducción prevista para alguno de los contaminantes -como es el caso de los óxidos de nitrógenopuede ayudar a paliar, en cierta medida, los problemas existentes en algunos puntos de la ciudad, en los que se superan en ocasiones los límites establecidos por la legislación vigente; éste es el caso de las zonas cercanas al Sur y Suroeste de la M-30.

Lógicamente, las zonas atravesadas por el nuevo cinturón reciben más efectos negativos que en la situación anterior, pero estas zonas están poco o nada pobladas y presentan características desde el punto de vista de la dispersión, mucho más favorables que las áreas urbanas: espacios abiertos, sin edificaciones, etc.

En definitiva, la modelización de la movilidad permite afirmar que se producirá un proceso de descongestión del cinturón de circunvalación $\mathrm{M}-30 \mathrm{y}$, sobre todo, del interior de la ciudad. Este fenómeno será particularmente acusado en lo relativo al tráfico de vehículos pesados, que sería canalizado en mayor medida por la M-40 cuando ésta se complete. Estos datos confirman los ya observados con la construcción de los tramos Sur y Este de dicha carretera, actualmente en servicio.

\section{Conclusiones}

El resultado de la evaluación de impactos realizada permite afirmar que la construcción del tramo Noroeste de la M-40 supone un impacto positivo, reduciéndose la congestión y disminuyendo consumos y emisión de gases contaminantes; éstos son máximos cuando el vehículo circula en régimen congestionado, con abundantes paradas y arrancadas.

En primer lugar, el consumo de combustible disminuye. Esto se debe a que la velocidad media de los vehículos aumenta: la circunvalación extrae parte del tráfico interior y lo traslada al exterior, facilitando así la dispersión de las emisiones.

En cuanto a la contaminación atmosférica, se puede destacar que las zonas en donde estos niveles de concentración disminuyen corresponden a áreas en las que hay población residente y que el tráfico y sus emisiones se trasladan a lugares con pocas o ningunas zonas residenciales.
La metodología restringida a los factores del medio considerados, ha permitido cuantificar los impactos, de forma que la comparación por diferencia entre la situación sin proyecto y cada alternativa puede realizarse fácilmente

\section{BIBLIOGRAFIA}

BERTRAND, T.M(1978): Congestion cost in a transport system Journal of Transportation Economics and Policy, 12(3), pp 244-279.

CARRASCO, C. et al. (1992): Leyes medio ambiente: Sistema Medio Ambiente. Tomo I: Parte General. Contaminación Atmosférica. Distribuciones La Ley, S.A. Madrid.

INECO (1989): Encuesta de movilidad 1987-1988. Consorcio Regional de Transportes. Madrid.

McCRAE (1988): Modelling the dispersion of vehicle-emitted pollutants. 81 st Meeting of APCA. Dallas, Texas

MONZÓN, A. et al. (1995): Valoración de los beneficios energéticos y medioambientales de las circunvalaciones urbanas. Inédito.

MOPT (1992): Carreteras Urbanas. Recomendaciones para su planeamiento y proyecto. Madrid.

MOPU(1992): Recomendaciones parala evaluacióneconómica, Coste-Beneficio de estudios y proyectos de carreteras. Actualización del valor del tiempo y costes de accidentes y combustibles. Servicio de Planeamiento. Madrid.

PASQUILLE \& SMITH; (1982): Atmospheric diffusion. 3rd Edic. Ellis Horwood, Chichester.

Proyecto PECAM (1991): Factores de emisión para el transporte en la Comunidad de Madrid. Módulo de Impacto Ambiental. Ref.: 31.07.IT18, Comunidad de Madrid.

VILLANUEVA, J. (1994): Metodologia de evaluación de los costes derivados de la congestión en las ciudades. Tesis Doctoral. E.T.S.I. de Caminos, Canales y Puertos. Universidad Politécnica de Madrid. 